\title{
Suppression of Optical Damage to the Surface of a High-Energy Q-Switched Ruby Laser Rod for Medical Use
}

\author{
Hiroyuki NISHIMURA, ${ }^{1}$ Kazuyoku TEI ${ }^{2,3}$ and Tomoo FUJIOKA ${ }^{2,3}$ \\ ${ }^{1}$ Japan Medical Equipment \& Consultant Co., Ltd. 3-31-3 Yushima, Bunkyo-ku, Tokyo 113-0034 \\ ${ }^{2}$ Department of Physics, Tokai University. 1117 Kitakaname, Hiratsuka-shi, Kanagawa-ken 259-1292 \\ ${ }^{3}$ Institute for Applied Optics. 2-5-5 Tomioka, Koto-ku, Tokyo 135-0047
}

(Received March 31, 2005)

\begin{abstract}
Optical damage to the surface of the ruby crystal rod in a Q-switched high-energy ruby laser for medical uses is analyzed, and a method to suppress the optical damage is successfully implemented.
\end{abstract}

Key Words: Ruby laser, Q-switched ruby laser, Optical damage, Method to suppress optical damage, Medical laser

\section{Introduction}

The ruby laser was invented by Maiman in 1960 as the first successful laser. Since then, ten of thousands of laser types have been invented and reported. However, in terms of practical use only a few laser types-produced in great numbers-are widespread.

The ruby laser is currently being applied in medicine in the treatment of macula on human skin. Because the ruby laser has been around the longest, most of problems with its operation have been solved and it has become a standard technology. However, at least one problem still remains with its application. For medical treatment of skin macula, the high-energy ruby laser should oscillate with stable short pulse. Also, the cost should be as low as possible.

To obtain high power, the ruby crystal needs to be large and requires high input power from the large flash lamps. Because of the large crystal diameter in high-energy storage, the distribution of absorbed energy in the crystal and the distribution of gain are not uniform in the rod volume, and are normally higher at the edges near the flash lamp.

When the gain is not uniformly distributed, the high-power ruby laser oscillates such that the distribution of light intensity is not uniform over the cross section of the oscillation. The light intensity in the high-gain areas is thus much higher than the value averaged over the cross section. This very strong light induces damage to the coating on the surface of the crystal.

The ruby laser is mostly used in the Q-switched mode to obtain regulated high peak power. As such, experimenters working on the high-power ruby laser have struggled with suppression of optical damage.

The optical damage also occurs in the bulk ruby crystal as well as on the surface. Kikin and Paramonov showed that the existence of pores in the crystal decreased the damage threshold in the crystal. ${ }^{1)}$ Denishchuk et al demonstrated that the beveled edge of the flat end of the ruby rod acted as a focusing surface and confirmed that the concentrated laser radiation caused local damage in the crystal rod. ${ }^{2)}$ Also, Kvapil et al have shown ex- perimentally that obtaining a ruby crystal with high optical quality by reducing inhomogeneity in the chromium concentration is important in increasing the damage threshold, ${ }^{3)}$ a result Danileiko et. al. have shown theoretically. ${ }^{4}$

Modern technologies have largely overcome the problem of optical damage in the ruby crystal rod. But optical damage to the ruby crystal surface is still an important problem.

Kikin first identified that a Fresnel diffraction maximum produced at the edges of the bulk defects caused damage to the end surface of the rod. ${ }^{5)}$ In other work, Danilenko et. al. showed the damage on the ruby surface is due to a thermal mechanism and the quality of the surface is an important factor. They also developed methods to clean the surface of the ruby rod to increase the damage threshold. $\left.{ }^{6}\right)$ Vrbova analyzed a passively Q-switched ruby laser and concluded the shortest pulse width should be $3 \mathrm{~ns}$ and the optical mode structure must be carefully controlled. ${ }^{7)}$ In late 1970s, the damage threshold of the surface of the ruby crystal was considered to be nearly $0.1 \mathrm{GW} / \mathrm{cm}^{2,7)}$ but one fourth century after that number increased 10 times by the technology progress.

The modern ruby laser used in medical applications should be of high energy and must have a highly multi mode structure. Technologies used to increase the optical damage threshold to the crystal surface have not yet been applied to a high-power, highly multimode ruby laser rod.

In the present work, we have successfully applied technologies to suppress optical damage in high-energy Q-switched ruby lasers.

\section{Summary of ruby lasers}

The ruby crystal is made of $\mathrm{Al}_{2} \mathrm{O}_{3}$ (sapphire) with some of the $\mathrm{Al}^{3+}$ ions replaced by $\mathrm{Cr}^{3+}$ ions, which are the origin of the pink color.

The outline of the energy structure of $\mathrm{Cr}^{3+}$ is shown in Fig. 1 . The light from the Xe flash lamp is absorbed in the two wide bands, the blue and green bands in the figure, and the absorbed energy in the two bands transits non radiatively to the two lasing 


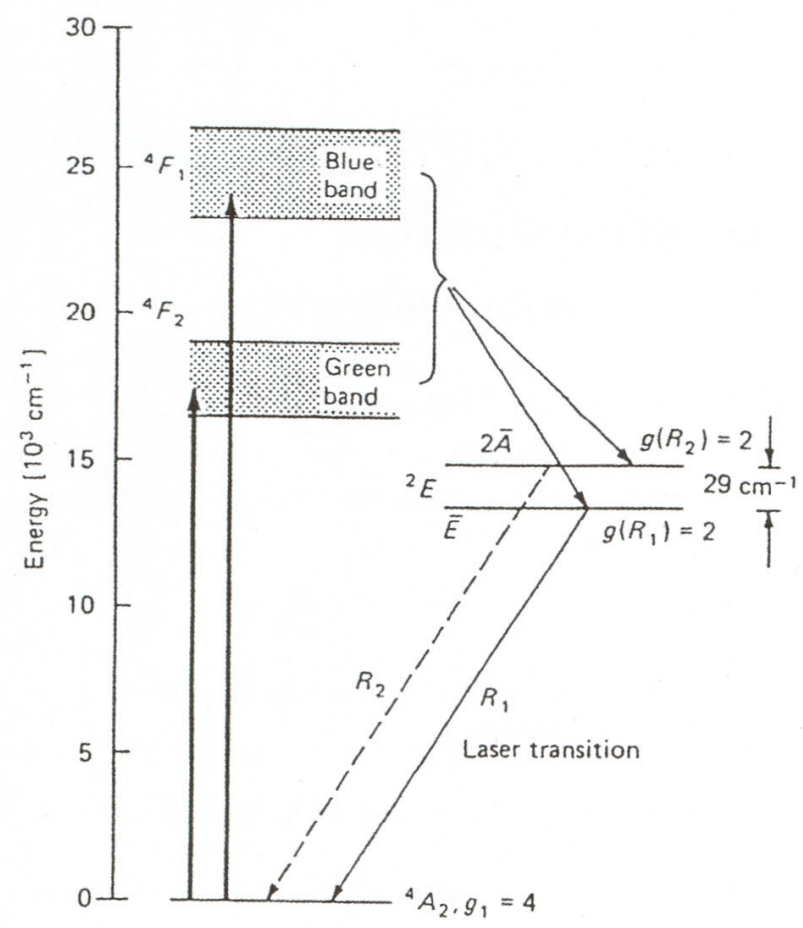

Fig. 1 Energy levels of $\mathrm{Cr}^{3+}$ in ruby.

bands, $2 \mathrm{~A}$ and $\mathrm{E}$, from which lasing occurs to the ground state. The lasing wavelengths of R1 and R2 are $694.3 \mathrm{~nm}$ and 694.9 $\mathrm{nm}$, respectively.

In the early stages, the Xe flash lamp used to excite the ruby crystal was a spiral shape, but straight flash lamps have since been used. In the laser, the ruby crystal rod and the flash lamp are fixed along the two focal lines of the elliptical pipe. Because high energy is required to get a high power output, two Xe flash lumps are normally used. When two Xe flash lamps are used, the two elliptical pipes are joined as shown in Fig. 2.

The small signal gain of the ruby laser is normally more than $0.2 / \mathrm{cm}$, and some times exceeds $0.28 / \mathrm{cm}$. This number indicates the ruby laser is a high gain laser and the oscillation occurs very easily. ${ }^{8}$ )

\section{Optical damage to the crystal surface and its suppression}

3.1 Damage to the antireflection coating on the surface of the ruby crystal

Figure 2 shows a cross section view of the ruby laser normally used in medical applications. The ruby crystal used in our experiments was $110 \mathrm{~mm}$ in length, $9.52 \mathrm{~mm}$ in diameter, and was excited by two Xe flash lamps, which consumed more than $1.6 \mathrm{~kJ}$ of electrical energy.

Two mirrors for the laser resonator are positioned opposite each other enclosing the ruby crystal, and a Pockel's cell and a polarizer are used to provide Q-switching.

At an input energy of $1.6 \mathrm{~kJ}$, the output energy of the laser is more than $7 \mathrm{~J}$ with a pulse width of $300 \mu$ s in the normal (free running) oscillation.

In the Q-switched mode, the output energy is up to $3 \mathrm{~J}$ with a 20 ns pulse width. However, at this power level the surface coating of the ruby crystal starts to become damaged after several shots. Even at an output energy of $1 \mathrm{~J}$, the edge of the surface become blackish after 200 shots, completely black after 250

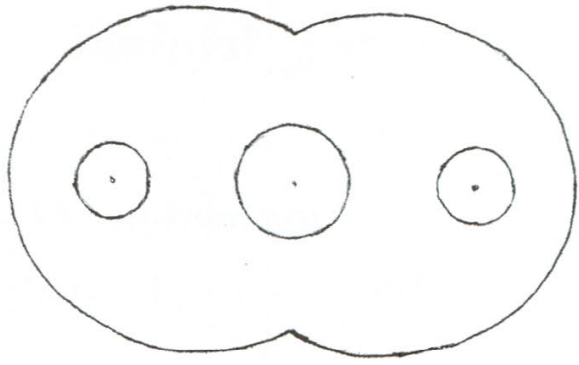

Fig. 2 Cross section of high-power ruby laser, the ruby rod (center) and the two flash lamps (both sides).

shots, and seriously damaged as shown in Fig. 3 after 300 shots, when two flat mirrors are used for the resonator.

Figure 4 shows the burn pattern of the ruby laser. The cross section of the oscillation is rectangular because the Pockel's cell and the polarizer in the cavity limit the oscillation area as shown in Fig. 4, the energy densities at both edges are much greater than in the center area.

At the side edge of the crystal, the distance to the flash lamp is the shortest and irradiation from the flash lamp is the highest-i.e. the laser gain is the highest. It is considered empirically that damage to the coating will occur at more than $1 \mathrm{GW} /$ $\mathrm{cm}^{2}$ laser irradiation. In our laser with a $2 \mathrm{~J}-20 \mathrm{~ns}$ laser output and a $0.7 \mathrm{~cm}^{2}$ surface area, the average peak power intensity on the surface is nearly $1 \mathrm{GW} / \mathrm{cm}^{2}$. And on the edge where the power intensity is much higher than the average, the peak intensity greatly exceeds the damage threshold.

3.2 Amplified spontaneous emission and the curvature of the mirrors used for the resonator

In a high-gain laser medium, the spontaneous emission can be amplified to produce partially coherent emission with a suitable level of power, allowing a reduction in gain for the main signal. ${ }^{9)}$ If $\alpha_{0} l$, the small signal gain times the gain length, is 1 , the amplification is 2.72 times; if $\alpha_{0} 1$ is 4 , the amplification exceeds 100 .

When amplified spontaneous oscillations occur in the nonsignal direction, the gain in the signal direction correspondingly reduces, which has sometimes been a very severe problem in

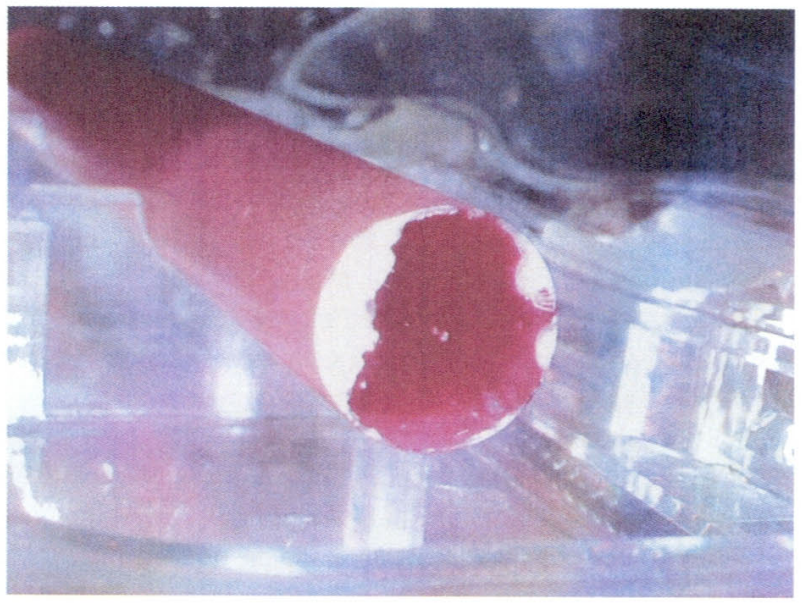

Fig. 3 Damage to the antireflection coating of the crystal surface after 300 shots at a $2 \mathrm{~J}$ output, without the groove. 


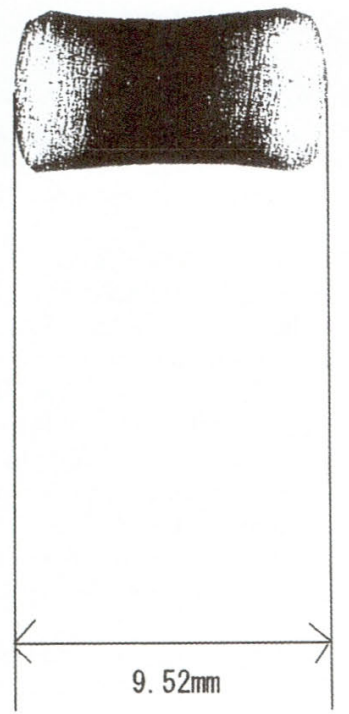

Fig. 4 Burn pattern induced by Q-switched ruby laser oscillation.

large Nd glass lasers and YAG lasers.

In our ruby laser, the average gain can be up to nearly $0.3 / \mathrm{cm}$, so, for a length of $11 \mathrm{~cm} \alpha_{0} l$ is nearly 3 , and at the edge can be very large.

When the reflectivity of the coated surface of the crystal is $0.1 \%$, the gain in the crystal rod exceeds the loss by 1000 times and oscillation occurs.

The amplified spontaneous emission is normally not a direct cause of the optical damage, but could contribute to it when using a blocking wire, which we mention later.

In the resonator of the ruby laser used in medical applications, a flat-flat mirror construction has mostly been used to produce a flat output power profile with high energy. In this construction, however, the flat mirror reflects the spontaneous emission to the coming direction and enhances the amplified spontaneous emission.

Instead of a flat mirror, we used a concave mirror of $30 \mathrm{~m}$ radius as a total reflection mirror. As a result, the damage threshold increased drastically, but damage still occurred at the edge of the surface after 200 shots with a Q-switched output of 2 J.

3.3 Technology to suppress optical damage at the edge areas To suppress optical damage of the coating on the ruby crystal surface, the laser oscillations with the highest gain should be inhibited.

In our experiment wire-like materials have been set in front of the edge areas in the resonator to block the strong oscillations to these areas. These wires have extended the life of the crystal surface, but the effect is not large. In our case, damage occurred at the edges of the crystal surface after 400 shots with a Qswitched output of $2.2 \mathrm{~J}$. The reason why blocking by the wire is not effective might be due to partial reflection at the wire surface. The partially reflected light from the ruby laser would be regenerated and could grow to a very high level. ${ }^{10}$ )

More effective blocking could be achieved by changes to the rod itself. P. Labudde et. al. proposed cutting a groove along the surface of the YAG crystal rod to suppress parasitic oscillation. ${ }^{11)}$ We tried the same method to reduce optical damage, and cut a groove of $0.3 \mathrm{~mm}$ radius and $0.6 \mathrm{~mm}$ pitch on the surface of the ruby crystal rod. The groove does not reduce absorption of radiation from the flash lamp or the total laser output energy. The existence of the surface groove was found to effectively suppress optical damage to the crystal surface; no damage was observed after more than 1000 shots with a $2.75 \mathrm{~J}-20$ ns output.

Figure 5 shows the results of the oscillation duration experiment. It is known that a short pulse oscillation at more than 2.5 $\mathrm{J}$ is adequate for the treatment of macula.

\section{Conclusion}

The ruby laser, the first successful laser developed, is currently in practical use in medical applications.

Despite its long history, the laser still has problems with its limited lifetime as a result of the high-energy laser output ( $>2$ $\mathrm{J} /$ pulse) and stable short pulse width (nearly $20 \mathrm{~ns}$ ) required.

Because there are no papers that tackle this problem for veryhigh-energy Q-switched ruby lasers, we investigated the origin

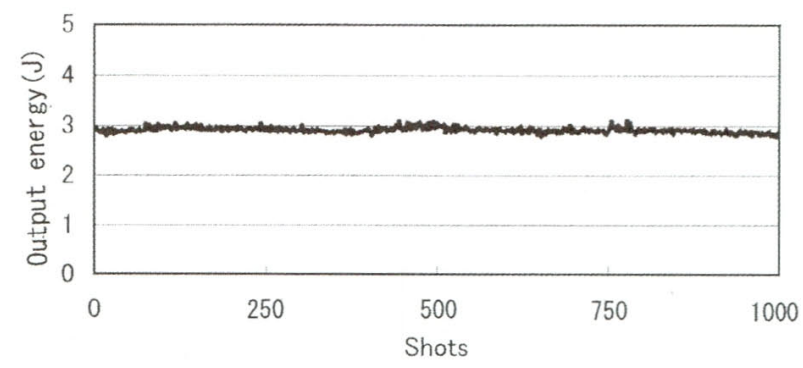

Fig. 5 1,000 shots operation of the ruby laser we developed with a groove at a pulse energy of more than $2.75 \mathrm{~J}$ with $\pm 6.5 \%$ stability.

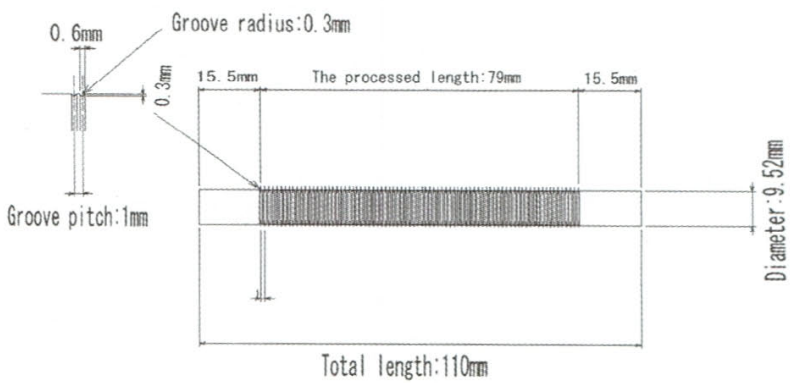

(a)

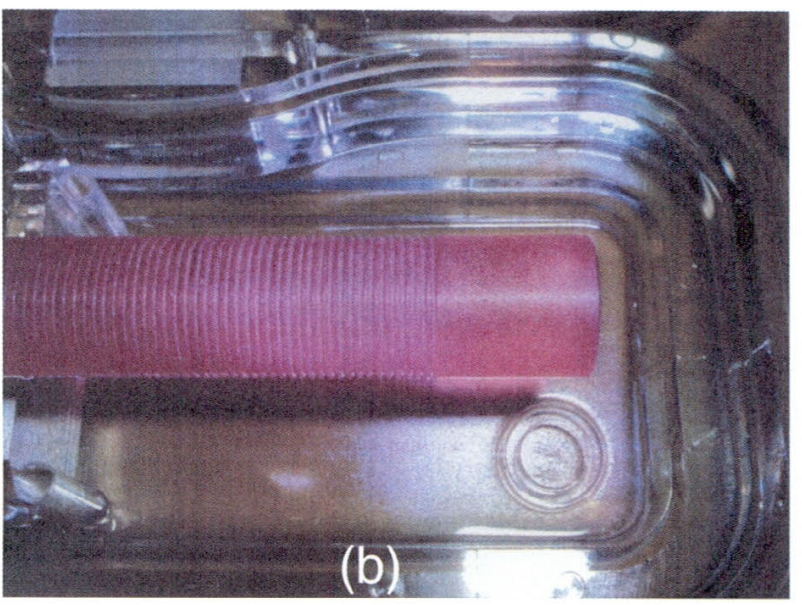

Fig. 6 Ruby laser crystal with grooves on the surface: a) dimensions and b) photograph of the crystal. 
of the short lifetime with regards to the very high gain at the edges of the crystal rod. By cutting a groove on the surface of the ruby rod, we succeeded in suppressing damage to the laser surface coating in a Q-switched ruby laser. A ruby laser operating at $2.75 \mathrm{~J} /$ pulse with a $20 \mathrm{~ns}$ pulse width operated for more than 1,000 shots without any damage, with $\pm 6.5 \%$ stability.

\section{Acknowledgements}

The authors would like to express hearty thanks to Messrs. Susumu Owada of Owada Optrodyne Inc., Kazuyuki Moro of Matsuo Sangyo Co., Ltd. and Satoshi Takaya of Japan Medical Equipment \& Consultant Co., Ltd. for assistance in preparing the experiments.

\section{References}

1) P. Yu Kikin and V. I. Paramonov: Sov. J. Quant. Electron 4 (1975) 1172.

2) V. V. Denishchuk, E. R. Dobrovinskaya, O. N. Kornilich, B. S. Perli, and B. S. Skorobogatov: Sov. J. Quant. Electron 7 (1977) 388.

3) J. Kvapil, B. Perner, and J. Kvapil: Czech. J. Phys. B28 (1978) 465.

4) Yu. K. Danileiko, A. A. Manenkov, A. M. Prokhorov, and V.

Ya. Khaimov-Mal'kov: J. Sov. Laser Reserch 1 (1980) 248.

5) P. Y. Kikin: Sov. J. Quant. Electron 5 (1976) 1283.

6) Yu. K. Danileiko, A. A. Manenkov, A. M. Prokhorov, and V. Ya. Khaimov-Mal'kov: J. Sov. Laser Reserch 1 (1980) 192.

7) M. Vrbova: IEEE J. Quant. Electron QE-14 (1978) 596.

8) W. Koechner: in Solid-State Laser Engineering (Fifth Revised and Updated Edition, Spriuger, 1999) 40.

9) R. C. Elton: X-ray lasers (Academic Press, Inc. (1990) p. 20.

10) P. Labudde, W. Seka, and H. P. Weber: Appl. Phys. Lett. 29 (1976) 732.

11) J. I. Davis and W.R. Sooy: Appl. Opt. 3 (1964) 715. 